\title{
Conductance Fluctuations in a Metallic Wire Interrupted by a Tunnel Junction
}

\author{
A. van Oudenaarden, M. H. Devoret,* E. H. Visscher, Yu. V. Nazarov, and J. E. Mooij \\ Department of Applied Physics and Delft Institute of Micro-electronics and Submicron-technology (DIMES), \\ Delft University of Technology, P.O. Box 5046, 2600 GA Delft, The Netherlands
}

(Received 14 January 1997)

\begin{abstract}
The conductance fluctuations of a metallic wire which is interrupted by a small tunnel junction has been explored experimentally. In this system, the bias voltage $V$, which drops almost completely inside the tunnel barrier, is used to probe the energy dependence of conductance fluctuations due to disorder in the wire. We find that the variance of the fluctuations is directly proportional to $V$. The experimental data are consistently described by a theoretical model with two phenomenological parameters: the phase breaking time at low temperatures and the diffusion coefficient. [S0031-9007(97)03081-0]
\end{abstract}

PACS numbers: 73.40.Rw, 72.15.Gd, 73.23.-b

Mesoscopic quantum interference phenomena in metallic wires, like weak localization and universal conductance fluctuations, are manifestations of the wave nature of electrons which are robust to microscopic disorder [1,2]. Changing the microscopic disorder of a mesoscopic metallic wire results in a change of the conductance of the order of the universal value $e^{2} / h$. Experimentally one generally measures the conductance fluctuations resulting from a change in the magnetic field, rather than from a change in the microscopic disorder. A large collection of experimental data from metallic to poorly conducting systems convincingly confirms the universal nature of the conductance fluctuations [3]. Although the influence of microscopic disorder is well understood, much less is known of the influence of the electron energy on the conductance fluctuations. In conventional experiments [3] on metallic wires it is very difficult to change the energy of the electrons in a controlled way. However, when the wire is interrupted by a tunnel junction it is possible to systematically examine the conductance fluctuations as a function of electron energy. The bias voltage over the wire drops almost completely inside the tunnel barrier, because the resistance of the tunnel junction is much larger than the resistance of the wire. The junction serves then as an injector of electrons in a specific energy range which can be tuned by the bias voltage. In this Letter we report on measurements of the conductance fluctuations in a metallic wire interrupted by a tunnel junction. We explored the dependence of the variance of the fluctuations on the bias voltage, magnetic field, and temperature. In particular, we measured autocorrelation functions to determine the correlation field and correlation voltage. We compare the full set of experimental data directly to theoretical predictions [4].

The fluctuations of the differential conductance across the wire + junction are due to interference of electron waves that return to the tunnel barrier after tunneling. In Fig. 1(a) such a trajectory is depicted. When an electron tunnels through the junction at point $a$ it diffuses through the right electrode and returns after numerous scat- ter events at point $b$. After tunneling to the left electrode at $b$ it diffuses back to point $a$. The dashed line in Fig. 1(a) denotes the trajectory of another electron wave which energy differs by $\varepsilon$ from the energy of the trajectory depicted by the full line. If the dephasing time $\tau_{\varphi}$ is smaller than $\hbar / \varepsilon$, the two partial waves propagate coherently and constructively interfere. An analogous interference effect occurs in a normal metal-superconductor tunnel junction. Here the subgap conductivity is determined by the interference of electron and hole waves

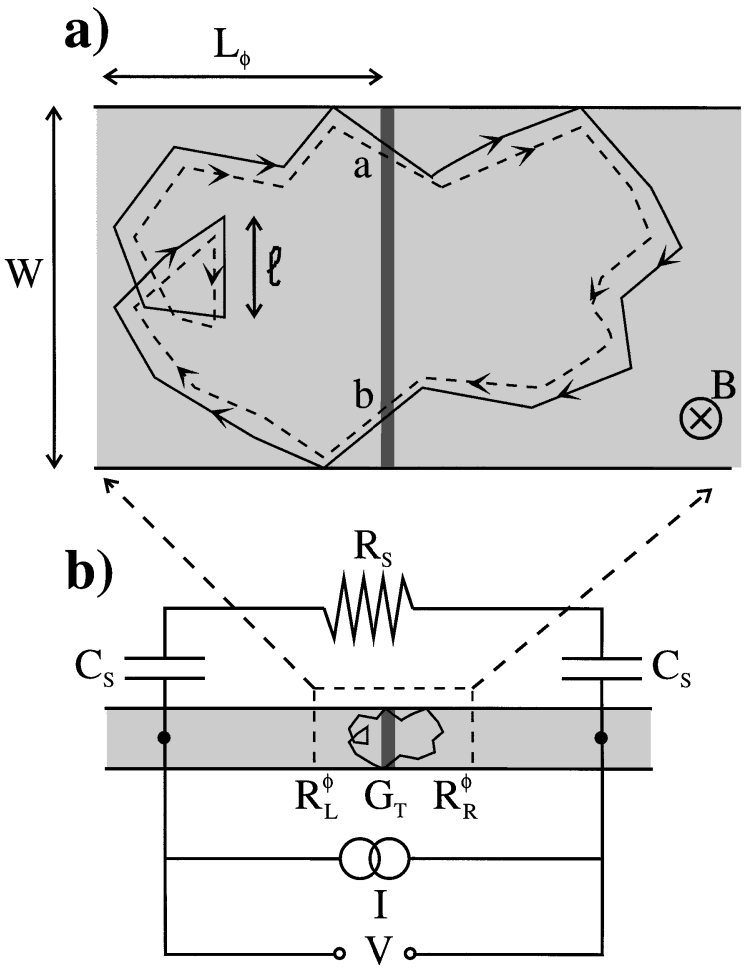

FIG. 1. (a) Class of trajectories which dominantly contribute to the conductance fluctuations. The direction of the magnetic field is perpendicular to the junction plane. (b) Electromagnetic environment of the diffusive wire and the tunnel junction. The light grey area denotes the diffusive wire and the dark grey area the tunnel barrier. 
returning to the junction $[5,6]$. The maximum length of the trajectory in Fig. 1(a) is $\Lambda=v_{F} \tau_{\varphi}$, where $v_{F}$ is the Fermi velocity. The dominant contributions to the conductance fluctuations are those caused by the type of trajectories depicted in Fig. 1(a) with length $\Lambda$ [4]. The diffusing electrons can coherently penetrate in the electrode over a distance $L_{\varphi}=\sqrt{D \tau_{\varphi}}$ [Fig. 1(a)], where $D$ is the diffusion coefficient. The finite dephasing time $\tau_{\varphi}$ results in an energy broadening of $\gamma=\hbar / \tau_{\varphi}$. The normalized variance of the conductance fluctuations is given by [4]

$$
\frac{\left\langle\delta G^{2}\right\rangle}{G_{T}^{2}} \approx \frac{e V}{\gamma^{3}} \Delta_{L} \Delta_{R},
$$

where $\Delta_{L}$ and $\Delta_{R}$ are the typical level spacing in the left and right electrodes, respectively, and $G_{T}$ is the tunneling conductance. The angle brackets denote an average over impurity configurations. The former equation can be rewritten in terms of the quantum conductance $G_{Q} \equiv e^{2} / \hbar$ and the effective resistances $R_{L}^{\varphi}$ and $R_{R}^{\varphi}$ of the left and right lead [7],

$$
\frac{\left\langle\delta G^{2}\right\rangle}{G_{T}^{2}} \approx \frac{e V}{\gamma} G_{Q}^{2} R_{L}^{\varphi} R_{R}^{\varphi} .
$$

The resistances $R_{L}^{\varphi}$ and $R_{R}^{\varphi}$ are formed by a part of the lead with length $L_{\varphi}$ [Fig. 1(b)]. In contrast with conductance fluctuations of a single wire the conductance fluctuations due to the leads of a single tunnel junction are nonuniversal. The variance $\left\langle\delta G^{2}\right\rangle$ is directly proportional to $e V / \gamma$, which is the number of energy slices with width $\gamma$ above the Fermi energy. Each energy slice fluctuates independently. When the bias voltage is increased, the number of fluctuating slices increases linearly. The presence of the tunnel junction opens the possibility to change the number of independently fluctuating slices in a controlled way. This is very difficult in a single metallic wire. Even though the resistances of the leads are typically $10^{4}$ times smaller than the tunnel resistance, the fluctuations due to the leads can be of the order of $1 \%$ of the total resistance. This phenomenon totally contradicts classical addition formulas for resistances and is a pure quantum interference effect. In order to accurately measure the conductance fluctuations the single junction has to be surrounded by a well defined low impedance environment. In this case the zero-bias anomaly is almost suppressed [8] and the fluctuations can be measured on a well characterized background.

The sample was fabricated using electron beam lithography and a multilayer process [9]. A schematic layout of the sample is shown in Fig. 1(b). A small $\mathrm{Al}-\mathrm{Al}_{2} \mathrm{O}_{3}-\mathrm{Al}$ tunnel junction was fabricated using a shadow evaporation technique. The junction capacitance, estimated from the overlap area of the junction, is $2 \mathrm{fF}$. The tunnel conductance $G_{T}$ is $57 \mu \mathrm{S}$. The small junction is shunted on chip by a capacitance $C_{S}$ and a resistance $R_{S}$. The shunt capacitor is a large parallel plate capacitor of
$200 \times 200 \mu \mathrm{m}^{2}$ with $75 \mathrm{~nm} \mathrm{SiO}$ as dielectric. The shunt capacitance, measured at room temperature, is approximately $45 \mathrm{pF}$. The shunt resistor is provided by a platinum strip between the capacitor plates. The resistance $R_{S}$, determined by a four terminal measurement at low temperatures, is $28 \Omega$. The left and right junction leads have widths $W_{L}$ and $W_{R}$ of 200 and $400 \mathrm{~nm}$, and thicknesses $t_{L}$ and $t_{R}$ of 25 and $40 \mathrm{~nm}$.

The measurements were performed in a dilution refrigerator. The measurement leads were filtered by means of $\mathrm{RC}$ and copper powder microwave filters at the temperature of the mixing chamber. At room temperature the leads were additionally filtered by feedthrough $\Pi$ filters. The sample was mounted in a microwave tight "box-in-a-box" construction at the temperature of the mixing chamber.

The differential conductance $G$ of the tunnel junction was measured with a lock-in technique. In Figs. 2(a) and 2(b) $G$ is plotted as a function of the bias voltage $V$. In Fig. 2(c) $G$ is shown versus the magnetic field $B$. Note that $B$ is large enough to drive the aluminum into the normal state. The conductance fluctuations as a function of both $V$ and $B$ are reproduced when the experiment is repeated. The raw-data differential conductance has a small minimum at zero voltage bias [not shown in Fig. 2(a)]. This zero-bias anomaly is due to inelastic tunnel events [8]. In our sample this zero-bias anomaly is controlled by the low impedance environment resistor $R_{S}$. To eliminate the effect of the zero-bias anomaly from the conductance fluctuations, we subtracted from the raw-data differential conductance a term proportional

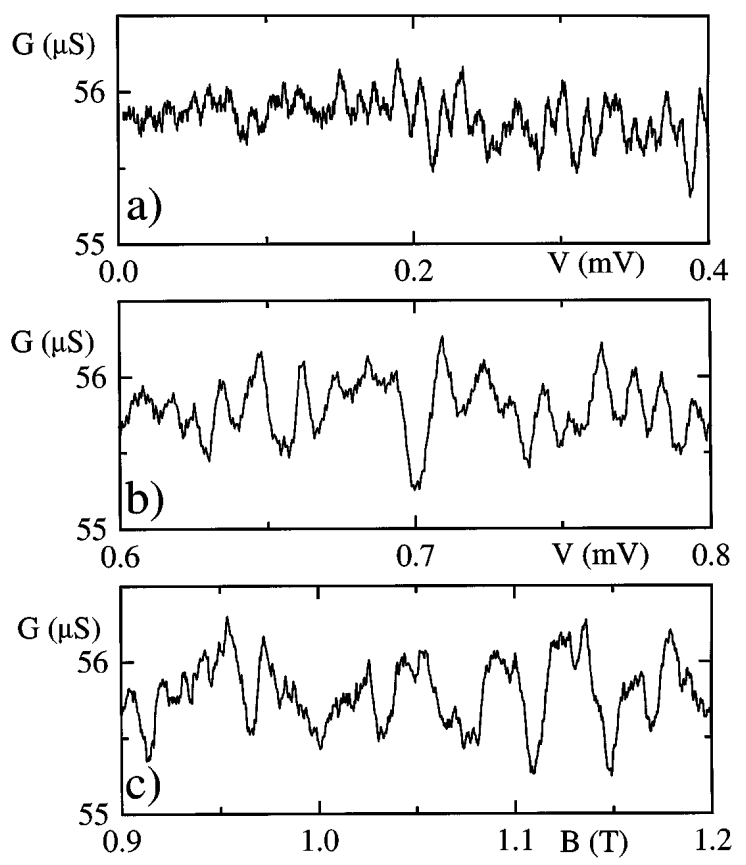

FIG. 2. (a) and (b) Differential conductance $G$ as a function of bias voltage $V$ at $T=20 \mathrm{mK}$ and $B=0.5 \mathrm{~T}$; (c) The differential conductance $G$ as a function of magnetic field $B$ at $T=20 \mathrm{mK}$ and $V=0.8 \mathrm{mV}$. 
to $V^{R_{S} G_{Q} / \pi}$. The result is shown in Figs. 2(a) and 2(b). The conductance fluctuations grow with increasing bias voltage. Changing the magnetic field is similar to changing the impurity configuration in the leads (ergodic hypothesis). By sweeping $B$ we get an effective ensemble average. We measured the variance $\left\langle\delta G^{2}\right\rangle$ using a data set of 5000 points, in which $G$ is measured from 0.2 to $1.7 \mathrm{~T}$ at a fixed bias voltage $V$. In Fig. $3\left\langle\delta G^{2}\right\rangle$ normalized to the square of the tunnel conductance $G_{T}$ is shown as a function of $V$ at different temperatures. For $V<0.8 \mathrm{mV}$ the normalized variance is directly proportional to $V$ as predicted by (2). At larger $V$ a saturation is observed probably due to out of equilibrium effects. In the inset of Fig. 3 the temperature dependence at $V=0.8 \mathrm{mV}$ is depicted.

The trajectory of a diffusing electron which returns to the tunnel barrier encloses, assuming Brownian motion, a typical area $\left\langle S^{2}\right\rangle^{1 / 2}=(1 / \sqrt{12}) L_{\varphi} W$, where $W$ denotes the electrode width [Fig. 1(a)]. When the magnetic field perpendicular to the electrode plane is changed by $\Phi_{o} /\left\langle S^{2}\right\rangle^{1 / 2}$ the two electron waves can no longer interfere constructively. Here $\Phi_{o}$ is the flux quantum $h / e$. A quantitative analysis can be done by extracting autocorrelation functions. In Fig. 4 the magnetoconductance correlation function $\langle\delta G(B) \delta G(B+\Delta B)\rangle$ normalized to the variance is plotted as a function of $\Delta B$. The correlation function is computed from a set of 5000 data points collected from 0.2 to $1.7 \mathrm{~T}$ at $V=0.8 \mathrm{mV}$ and $T=20 \mathrm{mK}$. In the inset of Fig. 4 the correlation function $\langle\delta G(V) \delta G(V+\Delta V)\rangle$ as a function of $\Delta V$ is plotted. This correlation function is calculated using 3000 data points from $V=-0.8$ to $V=0.8 \mathrm{mV}$. We eliminated the effect of the zero-bias anomaly as described be-

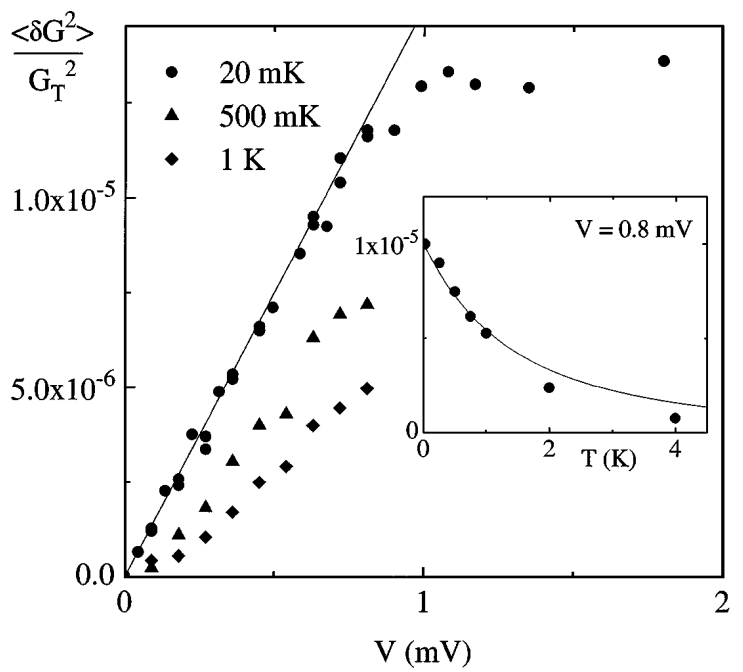

FIG. 3. Normalized variance as a function of $V$ for different temperatures. In the inset the normalized variance as a function of $T$ is shown at $V=0.8 \mathrm{mV}$. The full line in the inset denotes the theoretical result when Nyquist noise of the shunt resistor is the source of dephasing. fore. The full expression for the correlation functions is given by

$$
\begin{aligned}
\frac{\langle\delta G(V, B) \delta G(V+\Delta V, B+\Delta B)\rangle}{G_{T}^{2}} & \frac{4 V}{\pi e \hbar \nu_{L} \nu_{R} V_{m}^{2}} \int_{0}^{\infty} d t P_{\mathrm{cl}}^{L} P_{\mathrm{cl}}^{R} \cos \left(\frac{e}{\hbar} \Delta V t\right) \\
& \times e^{-2 t / \tau_{\varphi}} e^{-t / \tau_{B}} J_{1}^{2}\left(\frac{e}{\hbar} V_{m} t\right)
\end{aligned}
$$

Here $\nu_{L}$ and $\nu_{R}$ are the densities of states at the Fermi level in the left and right electrodes. The classical probability function $P_{\mathrm{cl}}^{L, R}(t)$ expresses the chance that an electron that leaves the barrier on the left or right side returns to the barrier in a time $t$. Assuming one-dimensional diffusion $\left(W \ll L_{\varphi}\right), P_{\mathrm{cl}}^{L, R}(t)=\left(S_{L, R} \sqrt{\pi D_{L, R} t}\right)^{-1}$, where $D_{L}$ and $D_{R}$ are the diffusion coefficients in the left and right electrodes and $S_{L}$ and $S_{R}$ the cross sections of the left and right electrodes.

The times $\tau_{\varphi}$ and $\tau_{B}$ describe the loss of coherence due to inelastic processes and due to the magnetic field, respectively. The dephasing time due to the magnetic field is given by $\tau_{B}=12(\hbar / e \Delta B)^{2} 1 /\left(D_{L} W_{L}^{2}+D_{R} W_{R}^{2}\right)$ [10]. The factor 2 in the exponent of Eq. (3) is due to the fact that both the left and right electrodes contribute a term $e^{-t / \tau_{\varphi}}$ to the integral. Another mechanism which provides a long-time cutoff is the finite instrumental resolution of the lock-in. The amplitude of the modulation voltage $V_{m}$ of the lock-in was $3 \mu \mathrm{V}$. In Eq. (3) this instrumental dephasing is governed by the square of the first order Bessel function of the first kind $J_{1}^{2}\left(\frac{e}{\hbar} V_{m} t\right)$.

The dephasing time $\tau_{\varphi}$ can be determined directly from the correlation function $\langle\delta G(V, B) \delta G(V+\Delta V, B)\rangle$. Using (3) we obtain $\tau_{\varphi}=289 \pm 10 \mathrm{ps}$ at $T=20 \mathrm{mK}$, which corresponds to an energy broadening $\gamma$ of $2.3 \mu \mathrm{eV}$. The fit result is shown as the full line in the inset of Fig. 4.

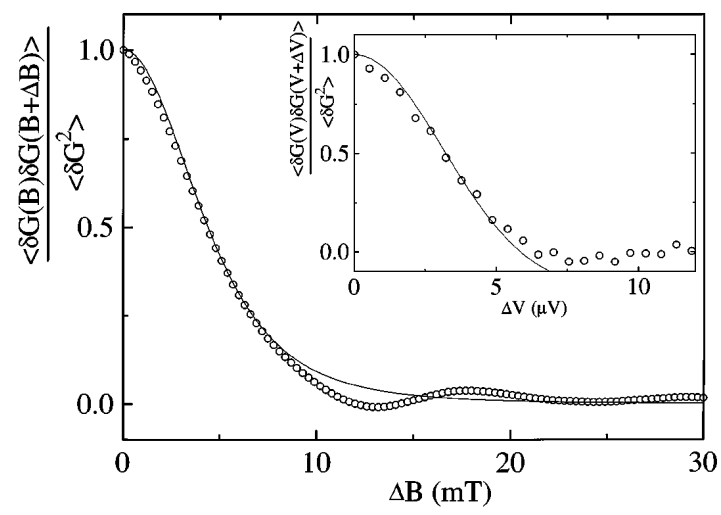

FIG. 4. Magnetoconductance correlation function at $T=20 \mathrm{mK}$ and $V=0.8 \mathrm{mV}$. The full line denotes the theoretical result with $D_{L}=0.0039 \mathrm{~m}^{2} / \mathrm{s}$ and $D_{R}=0.0063 \mathrm{~m}^{2} / \mathrm{s}$. In the inset the voltage correlation function is shown for $T=20 \mathrm{mK}$ and $B=0.5 \mathrm{~T}$. The full line represents the theoretical result for $\tau_{\varphi}=289 \mathrm{ps}$. 
The dephasing cannot be due to the Nyquist noise of the shunt resistor $R_{S}$. Nyquist noise would cause a dephasing time $\tau_{N}=\left[\left(\hbar / e^{2}\right) / R_{S}\right]\left(\hbar / k_{B} T\right)$ of $50 \mathrm{~ns}$ at $T=20 \mathrm{mK}$, which is more than two orders larger than $\tau_{\varphi}$. We therefore introduce a phenomenological dephasing time $\tau_{\varphi}(0)$, which accounts for dephasing at the lowest temperatures. At larger temperatures the effective dephasing time is then given by $\tau_{\varphi}^{-1}(T)=\tau_{\varphi}^{-1}(0)+\tau_{N}^{-1}(T)$. From $\langle\delta G(V, B) \delta(V, B+\Delta B)\rangle$ we can extract the diffusion coefficient $D_{L}=0.0039 \pm 0.0002 \mathrm{~m}^{2} / \mathrm{s}$ and $D_{R}=$ $0.0063 \pm 0.0002 \mathrm{~m}^{2} / \mathrm{s}$ (full line in Fig. 4), which are compatible with previous experiments [6]. Here we assumed that the ratio of the thicknesses $t_{L} / t_{R}$ equals $D_{L} / D_{R}$. Using these data we find a coherence length $L_{\varphi}$ of $1.2 \mu \mathrm{m}$ at $T=20 \mathrm{mK}$. The experimental values of the diffusion coefficient are consistent with $D=\frac{1}{3} v_{F} \ell$, where $\ell$ is the mean free path. Considering the thickness of the electrodes, $\ell$ is of the order of $10 \mathrm{~nm}$. When we use the free electron Fermi velocity $v_{F}=2 \times 10^{6} \mathrm{~m} / \mathrm{s}$ [11], we obtain $D \approx 0.007 \mathrm{~m}^{2} / \mathrm{s}$. The normalized correlation functions are determined with an accuracy of approximately $5 \%$. We calculate the variance at low temperatures using (3) and a density of states $\nu_{L}=\nu_{R}=$ $2.2 \times 10^{47} \mathrm{~J}^{-1} \mathrm{~m}^{-3}$, which is obtained from specific heat experiments [11]. For $T=20 \mathrm{mK}$ and $V=0.8 \mathrm{mV}$ we obtain $\left\langle\delta G^{2}\right\rangle / G_{T}^{2}=8 \times 10^{-6}$. This value is consistent with the experimental value $\left\langle\delta G^{2}\right\rangle / G_{T}^{2}=1 \times 10^{-5}$. We obtained the same results for another sample with $G_{T}=$ $62 \mu \mathrm{S}$. The variance at larger temperatures agrees with the predictions of (3) when Nyquist noise by a shunt resistor $R_{S}$ of $55 \Omega$ is taken into account (full line in the inset of Fig. 3). For $T>1 \mathrm{~K}$ the experimental values are smaller than the theoretical prediction assuming Nyquist noise. Probably at high temperatures other dephasing mechanisms start to play a role (e.g., electronelectron and electron-phonon interaction).

In summary, we have measured the conductance fluctuations in a diffusive wire which is interrupted by a tunnel barrier. The full set of experimental data can con- sistently be described by two phenomenological parameters, the residual phase breaking time and the diffusion coefficient. This last parameter is consistent with estimates of the mean free path. This type of experiments opens a new way of measuring the energy-dependent properties of diffusing electrons in disordered media.

We thank the Delft Institute of Micro-electronics and Submicron-technology (DIMES) for the support in fabrication. The financial support of the Dutch Foundation for Fundamental Research on Matter (FOM) is acknowledged.

*Permanent address: Service de Physique de l'Etat Condensé, CEA-Saclay, F-91191 Gif-sur-Yvette, France.

[1] Y. Imry, in Directions in Condensed Matter Physics, edited by G. Grinstein and G. Mazenko (World Scientific, Singapore, 1986), p. 100.

[2] B. L. Al'tshuler and A. G. Aronov, in Electron-Electron Interactions in Disordered Systems, edited by A. L. Efros and M. Pollak (North-Holland, Amsterdam, 1985).

[3] S. Washburn and R. A. Webb, Rep. Prog. Phys. 55, 1311 (1992).

[4] Yu. Nazarov, Zh. Eksp. Teor. Fiz. 98, 306 (1990) [Sov. Phys. JETP 71, 171 (1990)]; Yu. V. Nazarov, Physica (Amsterdam) 189B, 57 (1993).

[5] F. W. Hekking and Yu. Nazarov, Phys. Rev. Lett. 71, 1625 (1993); F. W. Hekking and Yu. Nazarov, Phys. Rev. B 49, 6847 (1994).

[6] H. Pothier, S. Guéron, D. Esteve, and M. H. Devoret, Phys. Rev. Lett. 73, 2488 (1994), and references therein.

[7] D. J. Thouless, Phys. Rev. Lett. 39, 1167 (1977).

[8] G.-L. Ingold and Yu. V. Nazarov, in Single Charge Tunneling, edited by H. Grabert and M.H. Devoret (Plenum, New York, 1992), Chap. 2.

[9] E. H. Visscher, S. M. Verbrugh, J. Lindeman, P. Hadley, and J. E. Mooij, Appl. Phys. Lett. 66, 305 (1995).

[10] C. W. J. Beenakker and H. van Houten, Phys. Rev. B 37, 6544 (1988).

[11] C. Kittel, in Introduction to Solid State Physics (John Wiley \& Sons, Inc., New York, 1986). 